\title{
Pearson's Correlation Coefficient: A More Realistic Threshold for Applications on Autonomous Robotics
}

\author{
Arthur de Miranda Neto \\ Federal University of Lavras, Lavras 37200-000, Brazil
}

\begin{abstract}
Many applications for control of autonomous platform are being developed and one important aspect is the excess of information, frequently redundant, that imposes a great computational cost in data processing. Taking into account the temporal coherence between consecutive frames, the PCC (Pearson's Correlation Coefficient) was proposed and applied as: discarding criteria methodology, dynamic power management solution, environment observer method which selects automatically only the regions-of-interest; and taking place in the obstacle avoidance context, as a method for collision risk estimation for vehicles in dynamic and unknown environments. Even if the PCC is a great tool to help the autonomous or semi-autonomous navigation, distortions in the imaging system, pixel noise, slight variations in the object's position relative to the camera, and other factors produce a false PCC threshold. Whereas there are homogeneous regions in the image, in order to obtain a more realistic Pearson's correlation, we propose to use some prior known environment information.
\end{abstract}

Key words: Perception, real time, mobile robots, Pearson’s correlation.

\section{Introduction}

In the last three decades, visual navigation for mobile robots or unmanned vehicles has become a source of countless research contributions. On the safety front, the progressive safety systems will be developed through the manufacturing of an "intelligent bumper" peripheral to the vehicle in answering new features as: blind spot detection, frontal and lateral pre-crash, etc. The objective in terms of cost to fill ADAS functions has to be very lower than the current adaptive cruise control (500 €) [1].

Machine vision is an important tool that continuously monitors the way forward, proving appropriate road information in real time. Aware that in the majority of the autonomous systems, the machine-vision system is working together with other sensors, added to its low cost, this work uses a monocular vision-based sensor. Because it uses simple

Corresponding author: Arthur de Miranda Neto, Ph.D. assistant professor, research fields: perception, localization, autonomous vehicles. E-mail: arthur.miranda@deg.ufla.br. techniques and fast algorithms, the system is capable to achieve a good performance, where the compromise between processing time and images acquisition is fundamental. Although extremely complex and highly demanding, thanks to the great deal of information it can deliver, machine vision is a powerful means for sensing the environment and it has been widely employed to deal with a large number of tasks in the automotive field.

The PCC (Pearson's Correlation Coefficient) [2] is widely used in statistical analysis, pattern recognition and image processing [3]. Based on Pearson's method, a visual-perception system based on an automatic image discarding method was proposed as a simple solution to improve the performance of a real-time navigation system by exploiting the temporal coherence between consecutive frames [4]. This idea is also presented in the key-frame selection technique [5]. Further, the PCC was applied as an environment observer method to save processor energy (power) consumption [6]. In the obstacle avoidance context for vehicles in dynamic and unknown environments, it has also proposed as a real-time collision risk 
estimation [7] and an extension of the environment observer method that selects automatically only the ROI (Regions-Of-Interest) [8].

To better understand this proposal, the Pearson's method is presented in Section 2, followed by the region-merging algorithm in Section 3 which aims to represent homogeneous regions. Finally, the conclusions are given in Section 4.

\section{Pearson's Correlation Coefficient}

The Pearson's method in Eq. (1) is widely used in statistical analysis, pattern recognition and image processing. Applications on the latter include comparing two images for image registration purposes, disparity measurement, etc. [3]. An example is given in Fig. 1.

$$
r_{1}=\frac{\sum_{i}\left(x_{i}-x_{m}\right)\left(y_{i}-y_{m}\right)}{\sqrt{\sum_{i}\left(x_{i}-x_{m}\right)^{2}} \sqrt{\sum_{i}\left(y_{i}-y_{m}\right)^{2}}}
$$

where is the intensity of the pixel in image 1 , is the intensity of the pixel in image 2, is the mean intensity of image 1 , and is the mean intensity of image 2 . The PCC threshold has value 1 if the two images are identical, 0 if they are completely uncorrelated, and -1 if they are completely anti-correlated, for example, if one image is the negative of the other.

\section{Region-Merging Algorithm}

The region-merging algorithm mainly aims to represent homogeneous regions. In this context, distortions in the imaging system, pixel noise, slight variations in the object's position relative to the camera, and other factors produce a PCC threshold value less than 1 , even if the object has not been moved or physically altered in any manner. Whereas there are homogeneous regions in the image, and in order to obtain a Pearson's correlation closer to reality, many proposed methods can be used, as for example, the OTM) (otsu thresholding method) [9] and the Canny edge detector [10]. Different methods could be used for this task, such as [11], [12].
Fig. 2a and 2e represent an interval equivalent to $1 \mathrm{~s}$ (a certain analysis window), namely the reference (first) frame and the current (last) frame of the series. This pair of frames was submitted to OTM and also to the Canny edge detector. The Canny edge detector results are presented in Fig. $2 \mathrm{~b}$ and 2f. Fig. 2c and $2 \mathrm{~g}$ present the Canny step 2: in this case, it initiates then analyzing from the slice closest to the vehicle, going from the bottom edge of the image until a first detected edge; from this point, the image pixels are classified as foreground (black).

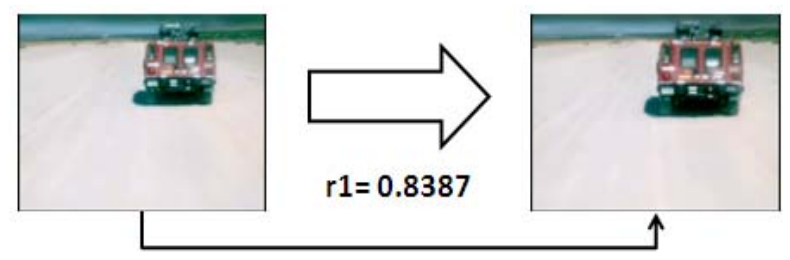

Fig. 1 Example of the PCC for two frames acquired in a deterministic interval.

(a)
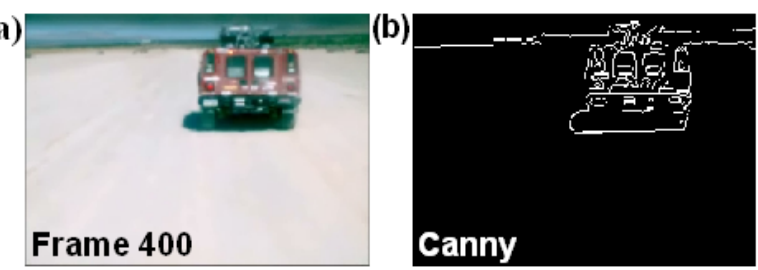

(c)
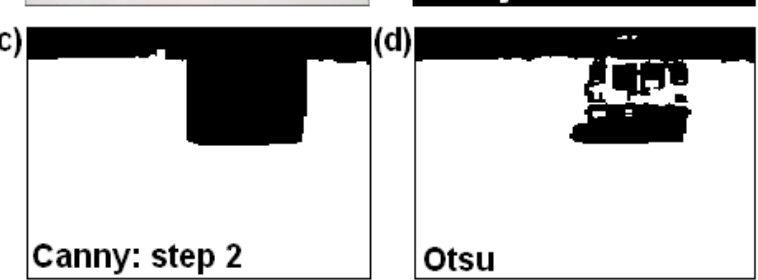

(e)
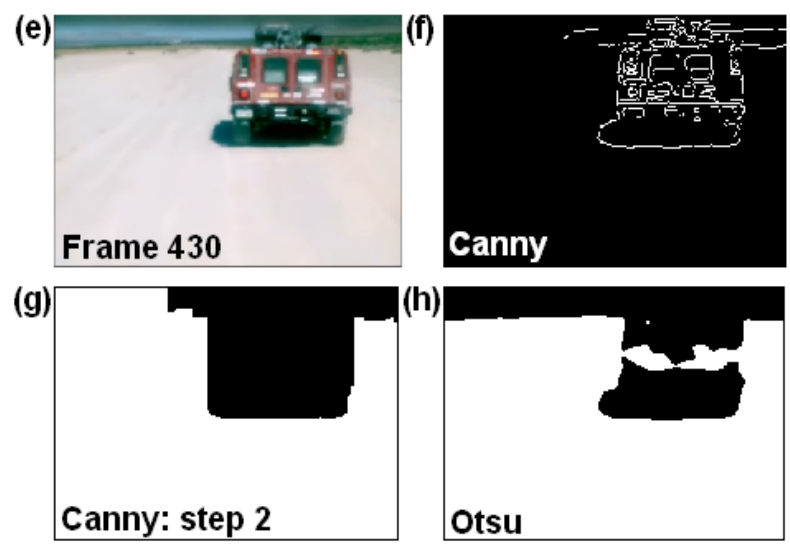

Fig. 2 DARPA Desert text-set [14]: (a) and (e) original frames; (b) and (f): the Canny edge detection; (c) and (g) are the binarized images by Canny step 2; (d) and (h) the binarized images by Otsu thresholding method. 
(a)
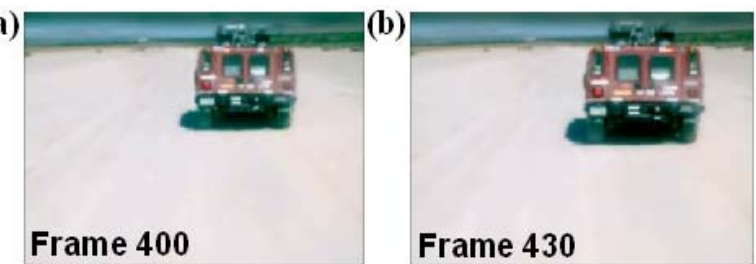

(c)

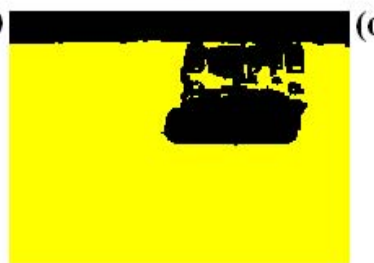

Frame 430
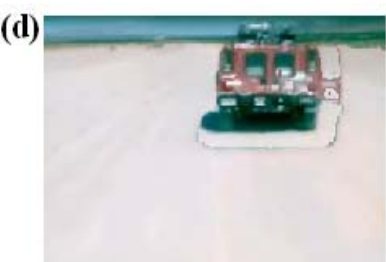

Fig. 3 DARPA Desert text-set [14]: (a) and (b) original frames; (c) binarized image by Otsu thresholding method and Canny step 2; (d) the Frame 400 with the background of the Frame 430.

(a)
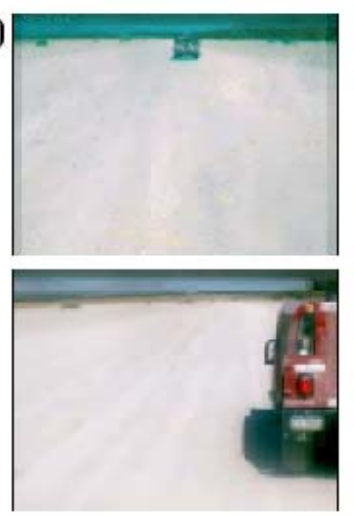

(b)

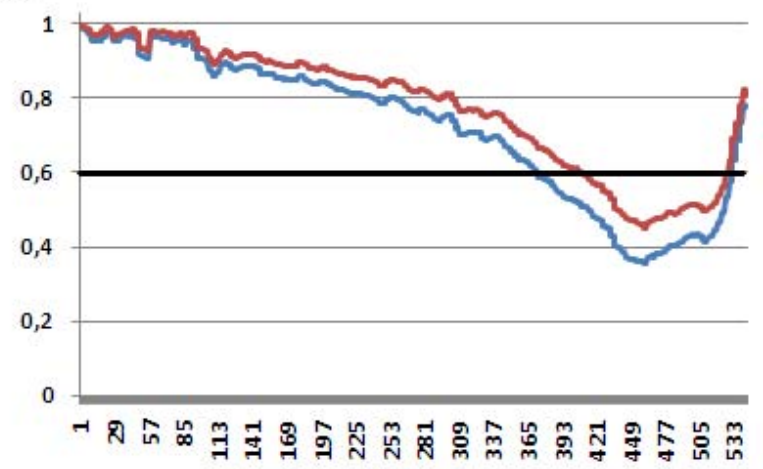

Fig. 4 DARPA Desert text-set [14]: (a) original frames; (b) from a reference frame (a): first frame, its correlation with all others; bue line: the Pearson's correlation; red line: the Pearson's correlation after the region-merging algorithm.

Finally, the OTM results are presented in Fig. 2d and 2h.

Right after these processes, the next step is shown in Fig. 3c, where it has a yellow pixel for each pair of background pixels in both binarized images, whether in OTM or Canny. These pixels represent the homogeneous regions that will be equaled. Then, the last step is shown in Fig. 3d): from this homogeneous region (yellow pixels) in Fig 3c), the pixels of the current frame will be copied to the reference frame, respectively: Fig. 3b and 3a. In resume, Fig. $3 d$ is the Fig. 3a with the background of the Fig. 3b. The Pearson's correlation Eq. (1) between Fig. 3a and 3b is 0.790 . After the region-merging algorithm, it was 0.800 between Figs. 3d and 3b Taken the DARPA Desert text-set [13], the new Pearson's correlation (Eq. (1)) results after the region-merging algorithm are presented in Fig. 4b: red line.

\section{Conclusion}

This work presents a simple solution to improve the performance of Pearson's correlation in order to obtain a more realistic threshold for applications on autonomous robotics. A remarkable characteristic of all methodologies presented here is its independence of the image acquiring system and of the robot itself. The same implementation can be used in different mobile robots and may be extended to other sensors. Futures work would also be focused to provide ground truth measurements from a front mounted radar and/or LIDAR system.

\section{References}

[1] Radio Spectrum Committee, European Commission, Public Document, Brussels, RSCOM10-35. December 02, 2010. http://ec.europa.eu/.

[2] Pearson, K. 1895. In Proceedings of Royal Society, 241-58.

[3] Eugene, Y. K., and Johnston, R. G. 1996. The Ineffectiveness of the Correlation Coefficient for Image Comparisons. Technical report LA-UR-96-2474, Los Alamos.

[4] Neto, A. M., Rittner, L., Leite, N., Zampieri, D. E., Lotufo, R., and Mendeleck, A. 2007. "Pearson's Correlation Coefficient for Discarding Redundant Information in Real Time Autonomous Navigation System.” IEEE Multi-conference on Systems and Control (MSC), Singapura.

[5] Wolf, W. 1996. "Key Frame Selection by Motion Analysis.” In Proceedings of the IEEE International 
Conference on Acoustics, Speech, and Signal Processing.

[6] Neto, A. M., Victorino, A. C., Fantoni, I., and Zampieri, D. E. 2011. "Real-Time Dynamic Power Management Based on Pearson's Correlation Coefficient.” Presented at the IEEE International Conference on Advanced Robotics, Tallinn, Estonia.

[7] Neto, A. M., Victorino, A. C., Fantoni, I., and Ferreira, J. V. 2013. "Real-Time Collision Risk Estimation Based on Pearson's Correlation Coefficient.” Presented at the IEEE Workshop on Robot Vision (WoRV), Florida, US.

[8] Neto, A. M., Victorino, A. C., Fantoni, I., and Zampieri, D. E . 2011. "Automatic Regions-of-Interest Selection Based on Pearson's Correlation Coefficient.” Presented at the IEEE International Conference on Intelligent Robots and Systems (IROS), ViCoMoR, California, US.
[9] Otsu, N. 1978. "A Threshold Selection Method from Gray-Level Histogram.” IEEE Transactions on Systems, Man, and Cybernetics. 8: 62-66.

[10] Canny, J. F. 1986. “A Computational Approach to Edge Detection.” IEEE Trans. Pattern Anal. Machine Intell. 6: 679-698.

[11] F. Bonin-Font, A. Ortiz, and G. Oliver, 2008. "Visual Navigation for Mobile Robots: A Survey." Journal of Intelligent and Robotic Systems 263-296.

[12] Chetan, J., Madhava, K., and Jawahar, C. V. 2010. “An Adaptive Outdoor Terrain Classification Methodology using Monocular Camera.” In Proceedings of the International Conference on Intelligent Robots and Systems, IROS.

[13] DARPA 2005. “DARPA Grand Challenge.” Accessed June 10, 2006. http://www.darpa.mil/grandchallenge05/ 